\title{
Reduction of drainage-associated complications in cardiac surgery with a digital drainage system: a randomized controlled trial
}

\author{
Arnaud Van Linden ${ }^{1}$, Florian Hecker ${ }^{1}$, Delphine S. Courvoisier ${ }^{2}$, Mani Arsalan ${ }^{1}$, Josepha Köhne ${ }^{3}$, \\ Christina Brei ${ }^{4}$, Tomas Holubec ${ }^{1}$, Thomas Walther ${ }^{1}$
}

${ }^{1}$ Department of Cardiothoracic and Vascular Surgery, Goethe University Hospital Frankfurt, Frankfurt, Germany; ${ }^{2}$ Faculty of Medicine, University of Geneva, Geneva, Switzerland; ${ }^{3}$ Department of Cardiac Surgery, Kerckhoff Clinic, Bad Nauheim, Germany; ${ }^{4}$ Medela Medizintechnik GmbH \& Co. Handels KG, Dietersheim, Germany

Contributions: (I) Conception and design: A Van Linden, T Walther; (II) Administrative support: A Van Linden, F Hecker, T Walther; (III) Provision of study materials or patients: A Van Linden, F Hecker, M Arsalan, J Köhne, C Brei, T Holubec, T Walther; (IV) Collection and assembly of data: A Van Linden, F Hecker, M Arsalan, J Köhne, T Holubec, T Walther; (V) Data analysis and interpretation: A Van Linden, DS Courvoisier, C Brei; (VI) Manuscript writing: All authors; (VII) Final approval of manuscript: All authors.

Correspondence to: Dr. Arnaud Van Linden. Department of Cardiothoracic and Vascular Surgery, Goethe University Hospital, Theodor-Stern Kai 7, 60590 Frankfurt, Germany. Email: arnaud.vanlinden@kgu.de.

Background: Thoracic chest drains are placed after cardiac surgery allowing for the clearance of blood, fluid, and air to prevent post-operative complications. Despite its importance, there is little data on the application of digital chest drainage systems in cardiac surgery. Therefore, the differences between an analog and a digital chest drainage system in cardiac surgery patients were investigated in a randomized controlled trial.

Methods: A total of 354 elective cardiac surgery patients were preoperatively randomized 1:1 between September 2016 and September 2017 to either an analog (Ocean) or a digital (Thopaz ${ }^{+}$) chest drainage system aiming to compare drainage-associated postoperative outcome parameters.

Results: A total of 340 patients were included in the analysis (analog: 188; digital: 152) with no significant differences in preoperative baseline parameters. Incidence of X-rays to detect air leaks was significantly lower in the digital group (analog: 20.2\%; digital: 8.6\%; $\mathrm{P}<0.01$ ). Patients treated with the digital system showed a $3.3 \%$ reduction of re-thoracotomies, however, not statistically significant (analog: 5.3\%; digital: 2.0\%; $\mathrm{P}=0.19)$. Median total fluid amount did not significantly differ between study groups [median (P25; P75); analog: 705 (400; 1,333) mL; digital: $686(404 ; 1,229) \mathrm{mL}$; $=0.83]$; however, the use of the digital drainage system resulted in a quicker removal with a reduced median drainage duration of 16 hours (analog: 65 hours; digital: 49 hours; $\mathrm{P} \leq 0.01$ ).

Conclusions: The study provides evidence that digital drainage systems can be safely applied in cardiac surgery patients. The use of the digital management system led to a decreased incidence of drainageassociated complications as well as to shortened chest tube duration. Findings require confirmation by additional studies.

Keywords: Digital chest drainage; cardiac surgery; drainage-associated complications

Submitted Jun 16, 2019. Accepted for publication Nov 19, 2019.

doi: $10.21037 /$ jtd.2019.12.20

View this article at: http://dx.doi.org/10.21037/jtd.2019.12.20 


\section{Introduction}

As a standard postoperative practice after cardiothoracic surgery, patients receive pleural and/or mediastinal chest drains to assist the clearance of blood, serous fluid, and air to prevent post-operative complications, such as cardiac tamponade (1), pleural effusion (2) or prolonged air leak (3).

Traditionally so-called "wet seal" drainage systems have been used. These collection containers have an integrated surge chamber and can be connected to external suction, usually integrated into the wall of the patient's rooms $(3,4)$. Disadvantages of analog systems are the patient's immobility and its subjective assessment of air leaks, which can lead to high interobserver variability in quantifying pulmonary air leak severity (5) and deciding when to pull the drain (6). For additional objective confirmation, $\mathrm{X}$-ray images are often used, which exposes the patient to radiation and incurs higher hospital costs (7).

For approximately 10 years, digital thoracic drains have been available on the market. A battery-operated mechanism provides a continuous and precisely controlled suction independent from external suction, and thus enables uninterrupted drainage from the operating room onwards (8). The significant advantages over traditional systems are the provision of objective data including trends shown numerically and in graphs displaying the progression of air leakage and fluid drainage measurement. This allows physicians to make clinical decisions with more precision. Besides, the monitored tubing with alarms presents the possibility of reduced tubing-related complications, such as chest tube blockage (9), while the portable device allows patients more mobility and independence in the postoperative phase (10).

While previous studies have mostly investigated differences between analog and digital drainage systems following thoracic surgery (11-16), there is little data published on the application of digital chest drainage systems in the cardiac surgery field comprising, to the best of our knowledge, merely conference abstracts from different studies (17-19).

Therefore, this prospective randomized controlled trial was designed to investigate potential differences in drainage-associated postoperative outcome parameters between an analog and a digital chest drainage system in cardiac surgery patients. As the primary outcome parameter, the incidence of chest $\mathrm{X}$-rays to detect an air leak was determined. Secondary outcomes included the incidence of re-thoracotomies due to tamponade or bleeding, the retention time of thoracic drains, the total amount of drained fluid, routine laboratory parameters, and length of hospital stay.

\section{Methods}

\section{Study design}

The current study had a prospective, 1:1 randomized, allcomer, single-center design for comparison of two chest drainage systems in cardiac surgery patients.

Baseline data included patient characteristics and blood samples. Routine pre-, intra- and postoperative in-hospital data were collected. A specific assessment of drainageassociated complications was performed.

The primary endpoint was the incidence of at least one chest X-ray with a clamped drain as detection of an air leak. Secondary endpoints were the incidence of re-thoracotomies due to tamponade or bleeding, retention time of thoracic drains, total amount of drained fluid at the time of chest drain removal, routine laboratory parameters (i.e., hemoglobin, leukocytes, creatinine), and length of hospital stay.

The enrollment and indexed procedures were performed between September 2016 and September 2017 at Kerckhoff Heartcenter, Bad Nauheim, Germany. All patients gave written informed consent, and the study was approved by the local ethical committee (Ethikkommission Landesärztekammer Hessen, Frankfurt). The ClinicalTrials. gov identifier number is NCT03021369.

\section{Patient inclusion and exclusion criteria}

All elective adult patients ( $\geq 18$ years of age) scheduled for cardiac surgery were screened for this study. There were no specific exclusion criteria.

\section{Randomization}

The study was designed for a 1:1 stratified randomization. The randomization was performed preoperatively after study inclusion, using a computer-based 'random number generator' (www.randomizer.org). Surgery was performed in a standard fashion and was not affected by the study protocol. The ethical committee demanded an interim analysis after 250 patients but allowed for further recruitment while the analysis was performed. After statistical analysis revealed that the significance of the 


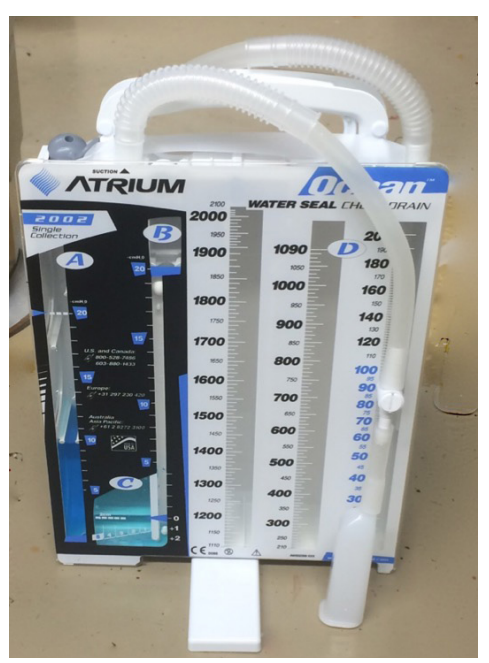

Figure 1 The analog drainage system Ocean Atrium (Maquet, Germany): a classical wet-seal drainage system with an analog scale to measure the total amount of fluid, a surge chamber and external vacuum-suction.

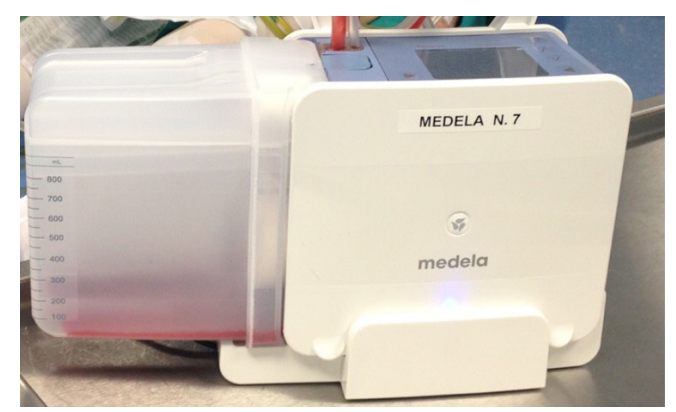

Figure 2 The digital drainage system Thopaz ${ }^{+}$(Medela, Switzerland): a digital drainage and monitoring system with onboard motor as vacuum source along with a suction control canister and water seal.

primary endpoint was reached, the recruitment was stopped with a study enrollment of 354 patients.

\section{Thoracic drainage systems}

The analog system was Atrium Ocean (Maquet, Germany). The Atrium Ocean is a classical wet-seal drainage system with an analog scale to measure the total amount of fluid, a surge chamber and external vacuum-suction (Figure 1).

The digital system was Thopaz (Medela, Switzerland), a compact, digital drainage, and monitoring system with an onboard motor as vacuum source along with a suction control canister and water seal (Figure 2). Due to its integrated sensors and software, the system provides automated management of prescribed suction while digitally tracking fluid output rates and air leak trends over time. The compact and lightweight design allows for early mobilization, even for patients on continuous suction. Alarms and notifications support troubleshooting on site. The device requires a power supply and must be charged regularly as it is advised in the instructions for use.

\section{Chest drainage assessment and clinical outcome}

Chest tubes were routinely placed by the surgeon depending on the type of surgery. A retrosternal polyvinyl chloride catheter (32 French) with side holes was placed in every patient undergoing cardiac surgery with sternotomy. Pericardial and/or pleural drains (24 or 28 French) were additionally placed if required. Single pleural drains were used in patients undergoing surgery with a lateral minithoracotomy. The drainage systems were connected to the drains in the operating room. The postoperative course did not vary from the clinical routine. Chest tubes were removed according to institutional standards, i.e., fluid output $<200 \mathrm{~mL} /$ day and no signs of air leak. The digital device quantifies and displays air leaks in $\mathrm{mL} / \mathrm{min}$, whereas air leak detection with the analog system requires visual detection via bubbling. An X-ray to detect air leaks has not routinely been performed, but only if there was suspicion of an air leak without clear signs, e.g., bubbling in wet seal. In such cases, the drains were clamped, and a chest X-ray was performed 2 hours later. The drains remained in the patients when the $\mathrm{X}$-ray showed a pneumothorax and were removed when a pneumothorax could be excluded. The clinical data on the fluid output, time of removal, air leaks, and tamponade were routinely recorded in a digital patient documentation system.

\section{Sample size calculation}

The calculation of sample size was based on the primary outcome. A decrease in the incidence of at least one chest $\mathrm{X}$-ray with a clamped drain to detect an air leak from $20 \%$ to $10 \%$ by using the digital chest drainage system is assumed. To detect a difference in proportions with $80 \%$ power using a significance level of 0.05 , a total of 394 patients were required. 


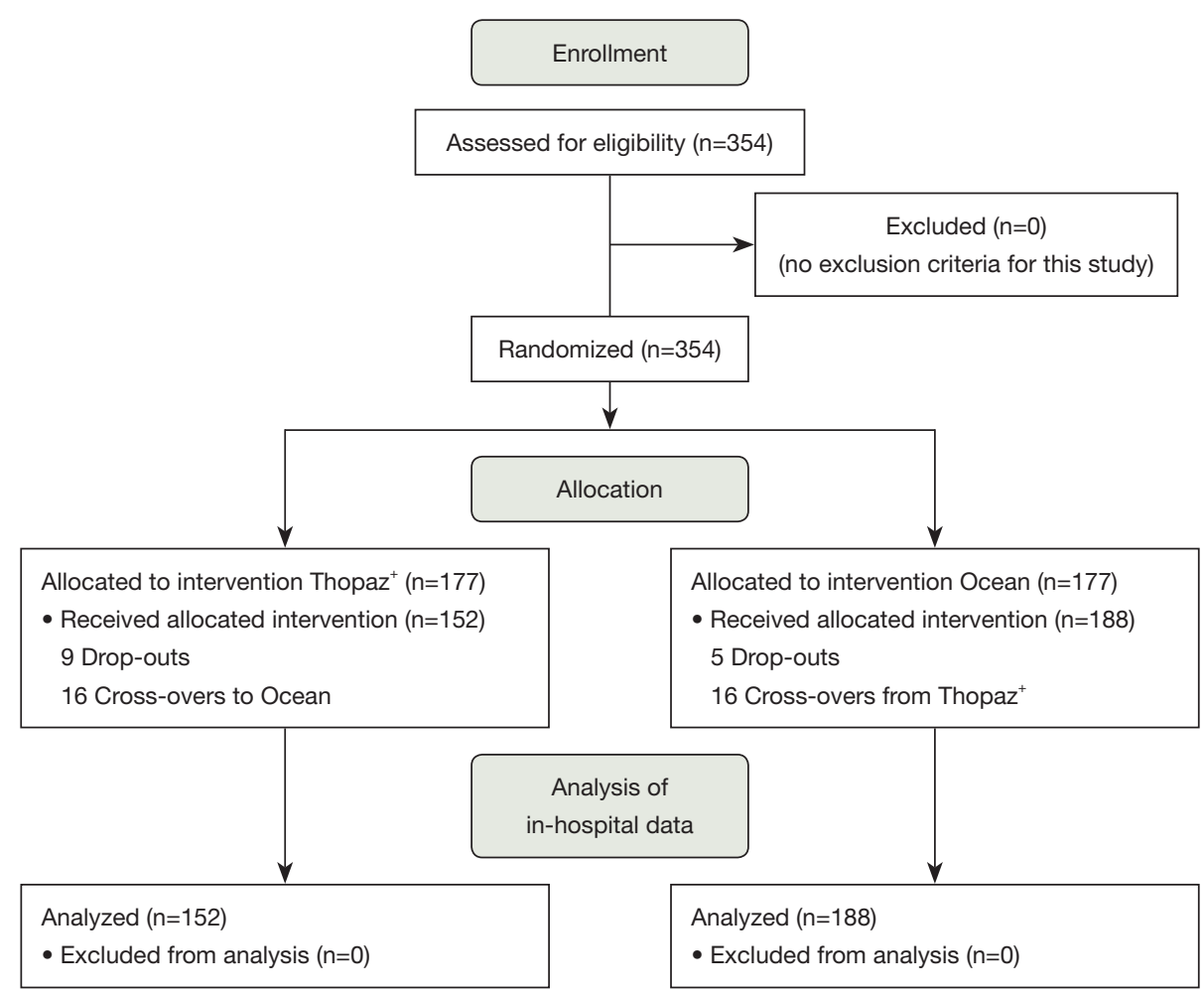

Figure 3 Flowchart of the enrollment, randomization and analysis of the study participants.

\section{Statistical analysis}

The analog and digital systems were compared using Fisher's exact test for categorical variables and Wilcoxon rank-sum test for continuous variables, since the distribution assessed by Kolmogorov-Smirnov test were often not normal. Per-protocol (PP) and intention to treat (ITT) analyses were carried out. All analyses were performed using R version 3.5.1 (R Foundation for Statistical Computing, Vienna, Austria) and SPSS Statistics software version 20 (IBM, Armonk, NY, USA), with a 2-sided $\mathrm{P}<0.05$ considered statistically significant.

\section{Results}

\section{Study population}

A total of 354 patients were assessed for eligibility and were randomized. There were 14 drop-outs in randomized patients due to surgery cancellation or undergoing interventional treatment. In the resulting cohort of 340 patients, there were 16 cross-overs, leading to 152 patients receiving a digital drainage system $\mathrm{Thopaz}^{+}$and 188 patients with the analog system Ocean. A flowchart of all participants is presented in Figure 3.

Since all drainage-associated outcome parameters are related to the actual device, we present the PP analysis. Additionally, ITT analyses were performed and can be found in a supplementary appendix online, without any significant differences to the $\mathrm{PP}$ results.

Of the 152 patients in the digital group, $74.3 \%$ were male and had a median age of $67.0(58.8 ; 74.0)$ years. The analog group was comparable with a male proportion of $70.2 \%$ with a median age of $67.0(58.0 ; 74.0)$ years. All baseline characteristics and intraoperative data are given in Tables 1 and 2 with no evidence of a difference between study groups. For both groups combined, in-hospital/30day mortality was $0.3 \%$ and the incidence of stroke was $0.9 \%$.

\section{Drainage-associated outcomes}

Postoperative data by intervention device are depicted in Table 3. Mean number of drains per patient was $2 \pm 0.8$ and median total amount of evacuated fluid was 705 and $686 \mathrm{~mL}$, respectively, with no evidence of a difference between both groups $(\mathrm{P}=0.83)$. The removal of drains was 
Table 1 Preoperative data by intervention device

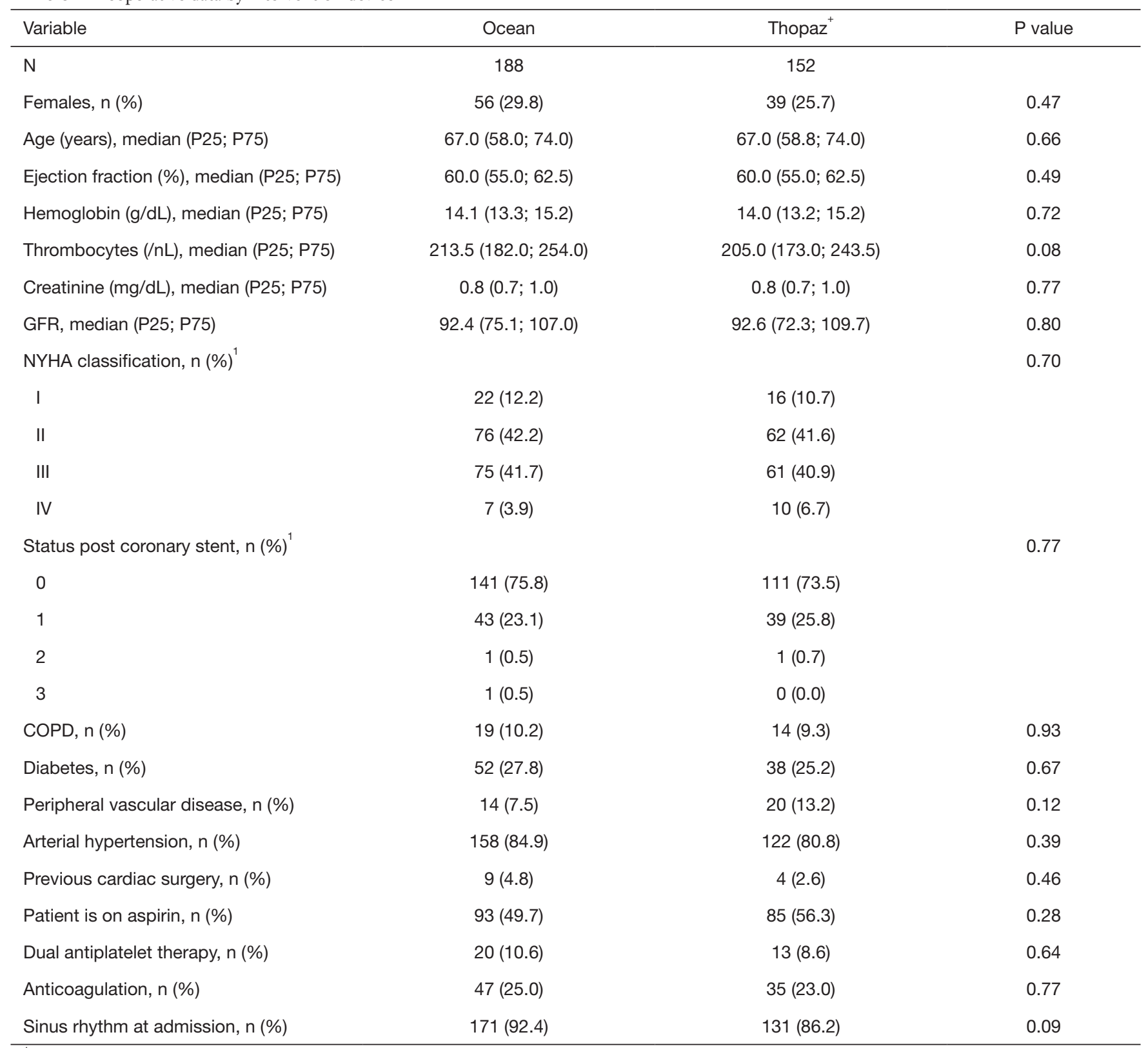

\footnotetext{
${ }^{1}$, due to some missing data, number of patients in each category may not sum to the total number of patients. NYHA, New York Heart
} Association; COPD, chronic obstructive pulmonary disease.

significantly earlier in the digital group (median, analog: 65 hours; median digital: 49 hours; $\mathrm{P} \leq 0.01$ ). Reduced chest tube duration had no significant effect on the length of ICU stay $(\mathrm{P}=0.57)$ or length of hospital stay $(\mathrm{P}=0.65)$ between the groups.

The incidence of chest $\mathrm{X}$-rays with clamped drains to detect air leaks was significantly lower for patients who received the digital chest drainage system (analog: 20.2\%; digital: $8.6 \% ; \mathrm{P}<0.01$, Figure 4). Reported as odds ratio $(\mathrm{OR})$ assessed using univariable logistic regression, this measure of association corresponds to an OR of 0.36 [95\% confidence interval (CI): 0.18-0.70, $\mathrm{P}=0.004]$. Adjusting for pre-surgery laboratory values and NYHA status, the OR was similar at 0.43 (95\% CI: $0.21-0.84, \mathrm{P}=0.02)$.

Within the same group, a lower proportion of rethoracotomies due to tamponade/bleeding was observed, 
Table 2 Intraoperative data by intervention device

\begin{tabular}{|c|c|c|c|}
\hline Variable & Ocean & Thopaz $^{+}$ & $P$ value \\
\hline Surgery, n (\%) & & & 0.83 \\
\hline Isolated CABG & $61(32.4)$ & $52(34.2)$ & \\
\hline Isolated AVR & $35(18.6)$ & $31(20.4)$ & \\
\hline Combined CABG \& AVR & $25(13.3)$ & $16(10.5)$ & \\
\hline Other & $43(22.9)$ & $30(19.7)$ & \\
\hline Aortic cross clamp time (min), median (P25; P75) & $62.0(49.0 ; 77.5)$ & $64.0(48.0 ; 85.8)$ & 0.36 \\
\hline Time of perfusion (min), median (P25; P75) & $100.0(78.5 ; 122.0)$ & $101.0(77.0 ; 129.0)$ & 0.54 \\
\hline Pleural drain right, $\mathrm{n}(\%)$ & $83(44.6)$ & $70(46.4)$ & 0.84 \\
\hline Substernal drain, n (\%) & $153(82.3)$ & $118(78.2)$ & 0.33 \\
\hline Pericardial drain, n (\%) & $44(23.7)$ & $39(26.0)$ & 0.71 \\
\hline
\end{tabular}

CABG, coronary artery bypass grafting; AVR, aortic valve replacement; $\mathrm{MV}$, mitral valve.

Table 3 Postoperative data by intervention device

\begin{tabular}{|c|c|c|c|}
\hline Variable & Ocean & Thopaz $^{+}$ & $P$ value \\
\hline Chest X-ray with clamped drain, $\mathrm{n}(\%)$ & $38(20.2)$ & $13(8.6)$ & $<0.01$ \\
\hline Re-thoracotomy due to tamponade/bleeding, n (\%) & $10(5.3)$ & $3(2.0)$ & 0.19 \\
\hline Chest X-ray with clamped drain or re-thoracotomy, $\mathrm{n}(\%)$ & $47(25.0)$ & $16(10.5)$ & 0.001 \\
\hline Total amount of fluid (mL), median (P25; P75) & $705(400 ; 1,333)$ & $686(404 ; 1,229)$ & 0.83 \\
\hline Need for new pacemaker, n (\%) & $7(3.8)$ & $10(6.7)$ & 0.34 \\
\hline Stroke, n (\%) & $2(1.1)$ & $1(0.7)$ & 1.00 \\
\hline In-hospital death, $\mathrm{n}(\%)$ & $1(0.5)$ & $0(0.0)$ & 1.00 \\
\hline
\end{tabular}


although statistical significance was not obtained (analog: 5.3\%; digital: $2.0 \% ; \mathrm{P}=0.19$ ). When both endpoints were combined as a safety endpoint, incidence of $\mathrm{X}$-rays to detect air leaks or re-thoracotomies due to tamponade/bleeding was significantly lower in the digital group (analog: 25.0\%; digital: $10.5 \% ; \mathrm{P}=0.001)$. Other parameters, such as the need for a new pacemaker, stroke, or in-hospital death did not differ between groups $(\mathrm{P}>0.05)$.

After the first three months, there was a trend towards fewer drain clamped $\mathrm{X}$-rays in the digital group (months

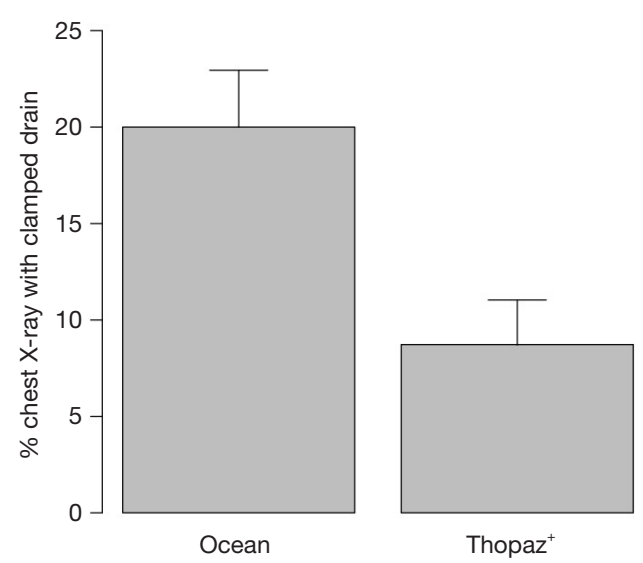

Figure 4 Significant reduction of chest $\mathrm{X}$-rays with clamped drains to detect air leaks in the digital Thopaz ${ }^{+}$drainage group $(20.2 \% v s$. $8.6 \%, \mathrm{P}<0.01)$.
1-3: $12.7 \%$; months 4-12: 4.1\%, $\mathrm{P}=0.08$ ).

Additional sensitivity analysis was performed and is given in Table 4. Data for analysis were restricted to patients with a pleural drain with very robust results observed.

\section{Discussion}

As part of post-surgical care, patients routinely receive at least one chest drain. Given that the presence of nonevacuated, retained blood are discussed in literature as severe $(20,21)$, it is surprising that little attention is directed toward improving drainage management methods. Typically, so-called "wet seal" drainage systems are used.

Herein, we report the results of a randomized controlled trial, which compared a digital drainage system (Thopaz; Medela, Switzerland) with a classic analog wet-seal system (Atrium Ocean; Maquet, Germany) in elective cardiac surgery patients in a German hospital. In the digital group, drainage-associated complications were significantly decreased, when looking at the incidence of $\mathrm{X}$-rays with clamped drain or re-thoracotomies due to tamponade/ bleeding. Besides, analyses indicated significantly shortened drainage duration with no differences in the total fluid amount at the time of chest drain removal between groups. Further postoperative outcome parameters did not significantly differ when study groups were compared.

The overall results of this study demonstrate the

Table 4 Sensitivity analysis of postoperative data with patients, who had a pleural drain inserted by intervention device

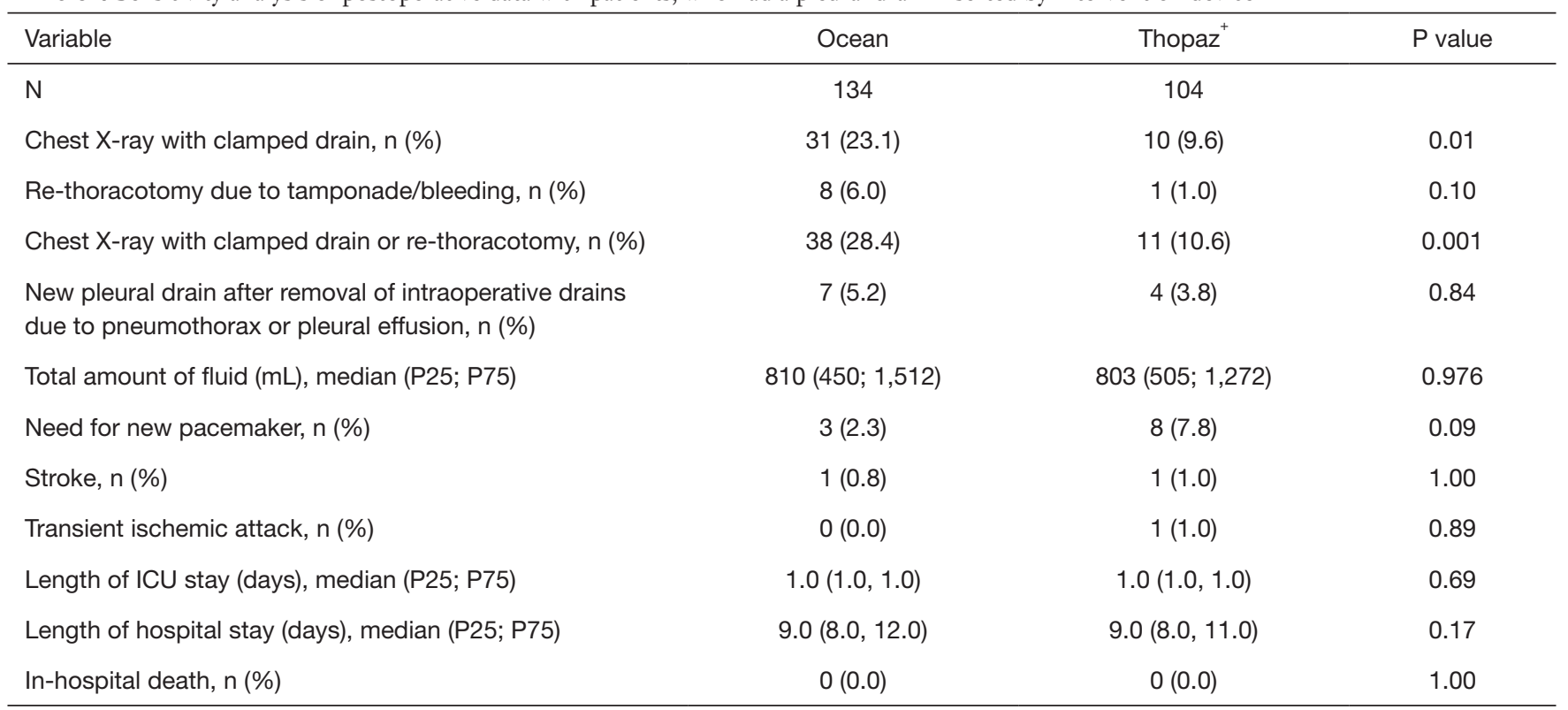


safety and efficacy of a digital drainage system (Thopaz $\left.{ }^{+}\right)$ in cardiac surgery patients in addition to a reduction in drainage-associated complications. The primary endpoint of the study was the incidence of chest $\mathrm{X}$-rays with clamped drains as a parameter for detecting air leaks. The need for X-rays with clamped drains was significantly lower in the digital group with an incidence of $8.6 \%$ compared to $20.2 \%$ in the analog group $(\mathrm{P}<0.01)$. This superiority in detecting air leaks confirms the findings of previous studies, demonstrating improved interobserver reliability $(5,6,13)$. In our study, there were still a number of patients in the digital group that underwent chest $\mathrm{X}$-ray with clamped drain. This might be explained by the institutional routine processes. Wet-seal systems were the standard systems for many years whereas digital devices were introduced shortly before the study started. Thus, the learning curve in handling the digital devices took place during the study period, which could explain the trend towards less need for drain clamped $\mathrm{X}$-rays in the digital group after three months of application (months 1-3: 12.7\%; months 4-12: $4.1 \%, \mathrm{P}=0.08)$. This might further explain why 16 patients were accidentally allocated to the analog group.

Associated with an increased postoperative complication rate and mortality, chest re-exploration in the early postoperative phase is a possible event of patients undergoing cardiac surgery. Consistent with current literature $(22,23)$, our results showed re-thoracotomy rates of $5.3 \%$ and $2.2 \%$, respectively. Without reaching significance, we could demonstrate a 3.3-percent reduction in the re-thoracotomy rate when the digital drainage system was used. One advantage of the digital device is the immediate start of drainage, enabling uninterrupted vacuum to the drains from the operating room onwards. It could be hypothesized that continuous suction evacuates more of the initial intraoperative blood that could potentially have, besides other factors, an impact on early pericardial tamponade.

Furthermore, a trend towards a decrease in new pleural drains after removal of the intraoperative drains was observed. The study, however, was not powered for detecting this difference with its low incidence.

So far, existing studies in the thoracic field have shown a reduced duration of chest tube placement and length of hospital stay when these digital devices were used $(13,14,16,24,25)$, resulting in significantly lower hospital costs (24). Study results were confirmed by a meta-analysis from Zhou and colleagues comparing prospective data of 10 randomized controlled trials from 1,268 patients undergoing lung resection surgery (26). Recently, the use of digital drainage systems has been recommended as part of the enhanced recovery after surgery (ERAS) pathway for the perioperative management after lung surgery in order to improve patients' outcome (27).

As the first prospectively randomized trial evaluating this topic in the cardiac field, our study was able to confirm the shorter duration of chest drains in cardiac patients with a reduced median drainage duration of 16 hours. Whereas in thoracic surgery the duration of the drain is considered the most critical influencing factor for the length of patient's hospitalization (28), cardiac surgery patients in Germany typically stay at least 1 week in the hospital and then are transferred directly to rehab. This may explain why the earlier removal of drains in the digital group did not result in shorter hospital stay in the current analysis. However, early postoperative mobilization has been shown to be an essential element to prevent postoperative complications, enhance functional capacity, and shorten the length of hospital stay (29). Considerable advantages of the digital drainage system are its portability and earlier removal of the drains. This could have major implications for postoperative recovery and rehabilitation efforts in patients, including greater opportunities to engage in physical activity and a risk reduction in sternal wound infections (30).

\section{Strengths}

Although it is the first study to compare digital and analog chest drainage systems in cardiac surgery patients, the strengths are the prospective randomized design and the all-comer setup. Different types of cardiac surgery need different number and types of chest drains and a selected potential biased setup might not represent 'real-world' data. The data collection included relevant parameters, such as air leak detection, amount of fluid, tamponade, duration of the drains, and need for new drains. Although there are institutions with other protocols to detect air leak besides $\mathrm{X}$-rays with clamped drains, the incidence of these $\mathrm{X}$-rays is a good parameter for statistical analyses. It can easily be measured and compared.

\section{Limitations}

This study was limited by the protocol deviation, leading to 16 cross-overs, randomized in the digital group, but were accidentally allocated to the analog group. Major efforts were made to train staff de novo to avoid future 
deviations. Hence, PP and ITT analyses were reported for all outcomes, with no significant differences between both analyses. With consideration of the study design, physicians were not blinded. The decision to perform an X-ray to detect air leak was conscientiously taken by the physicians, which may have introduced bias.

Due to a lack of comparative studies, additional randomized controlled trials with a greater variety of parameters will be needed to provide more definitive evidence and develop a full picture. Especially rethoracotomies due to early tamponade are rare, and a more significant number of patients are needed to find potential differences for this endpoint.

Economics of health care departments and cost management are important concerns for hospital administrations, thus further research is needed to analyze the cost-effectiveness of a digital versus an analog device.

\section{Conclusions}

The data provides evidence that digital drainage systems can be safely applied in cardiac surgery patients. Compared to the analog system, the application of the digital drainage system demonstrated a significantly decreased incidence of drainage-associated complications and shortened chest tube duration.

\section{Acknowledgments}

This work was supported by Medela Switzerland by providing the disposable containers.

\section{Footnote}

Conflicts of Interest: Arnaud Van Linden is consultant for Medela. Christina Brei is an employee of Medela Medizintechnik GmbH \& Co. Handels KG and gave scientific input during manuscript preparation. The other authors have no conflicts of interest to declare.

Ethical Statement: The authors are accountable for all aspects of the work in ensuring that questions related to the accuracy or integrity of any part of the work are appropriately investigated and resolved. All patients gave written informed consent, and the study was approved by the local ethical committee (Ethikkommission Landesärztekammer Hessen, Frankfurt). The ClinicalTrials. gov identifier number is NCT03021369.

\section{References}

1. Carmona P, Mateo E, Casanovas I, et al. Management of cardiac tamponade after cardiac surgery. J Cardiothorac Vasc Anesth 2012;26:302-11.

2. Labidi M, Baillot R, Dionne B, et al. Pleural effusions following cardiac surgery: prevalence, risk factors, and clinical features. Chest 2009;136:1604-11.

3. Baringer K, Talbert $\mathrm{S}$. Chest drainage systems and management of air leaks after a pulmonary resection. J Thorac Dis 2017;9:5399-403.

4. Satoh Y. Management of chest drainage tubes after lung surgery. Gen Thorac Cardiovasc Surg 2016;64:305-8.

5. McGuire AL, Petrcich W, Maziak DE, et al. Digital versus analogue pleural drainage phase 1: prospective evaluation of interobserver reliability in the assessment of pulmonary air leaks. Interact Cardiovasc Thorac Surg 2015;21:403-7.

6. Varela G, Jiménez MF, Novoa NM, et al. Postoperative chest tube management: measuring air leak using an electronic device decreases variability in the clinical practice. Eur J Cardiothorac Surg 2009;35:28-31.

7. Cunningham JP, Knott EM, Gasior AC, et al. Is routine chest radiograph necessary after chest tube removal? J Pediatr Surg 2014;49:1493-5.

8. Cerfolio RJ, Varela G, Brunelli A. Digital and Smart Chest Drainage Systems to Monitor Air Leaks: The Birth of a New Era? Thorac Surg Clin 2010;20:413-20.

9. Rathinam S, Bradley A, Cantlin T, et al. Thopaz Portable Suction Systems in Thoracic Surgery: an end user assessment and feedback in a tertiary unit. J Cardiothorac Surg 2011;6:59.

10. Danitsch D. Benefits of digital thoracic drainage systems. Nurs Times 2012;108:16-7.

11. Lijkendijk M, Licht PB, Neckelmann K. Electronic versus traditional chest tube drainage following lobectomy: a randomized trial. Eur J Cardiothorac Surg 2015;48:893-8; discussion 898.

12. Brunelli A, Salati M, Refai M, et al. Evaluation of a new chest tube removal protocol using digital air leak monitoring after lobectomy: a prospective randomised trial. Eur J Cardiothorac Surg 2010;37:56-60.

13. Cerfolio RJ, Bryant AS. The Benefits of Continuous and Digital Air Leak Assessment After Elective Pulmonary Resection: A Prospective Study. Ann Thorac Surg 2008;86:396-401.

14. Pompili C, Detterbeck F, Papagiannopoulos K, et al. Multicenter international randomized comparison of objective and subjective outcomes between electronic 
and traditional chest drainage systems. Ann Thorac Surg 2014;98:490-6; discussion 496-7.

15. Filosso PL, Ruffini E, Solidoro P, et al. Digital air leak monitoring after lobectomy for primary lung cancer in patients with moderate COPD: can a fast-tracking algorithm reduce postoperative costs and complications? J Cardiovasc Surg (Torino) 2010;51:429-33.

16. Jablonski S, Brocki M, Wawrzycki M, et al. Efficacy assessment of the drainage with permanent airflow measurement in the treatment of pneumothorax with air leak. Thorac Cardiovasc Surg 2014;62:509-15.

17. Barozzi L, Biagio LS, Meneguzzi M, et al. Do We Still Need Wall Suction for Chest Drainage? Heart Lung Circ 2018;27:S502.

18. Hofmann S, Awad G, Asch S, et al. The Influence of Dynamically Regulated, Intermittently Regulated Active Chest Drainage Units Compared to Conventional Vacuum-Driven Systems on the Early Postoperative Outcome after Cardiac Surgery. Thorac Cardiovasc Surg 2018;66:S1-110.

19. Mrowczynski W, Kurzynska P, Henschke J, et al. Portable Digital Chest Drainage System in Pediatric Patients Undergoing Fontan Procedure. Geneva, 2014.

20. Boyle EM Jr, Gillinov AM, Cohn WE, et al. Retained Blood Syndrome After Cardiac Surgery. Innovations (Phila) 2015;10:296-303.

21. St-Onge S, Perrault LP, Demers P, et al. Pericardial Blood as a Trigger for Postoperative Atrial Fibrillation After Cardiac Surgery. Ann Thorac Surg 2018;105:321-8.

22. Dimberg A, Alstrom U, Janiec M. Re-exploration for bleeding associated with increased incidence of the need for reintervention after coronary artery bypass graft surgery. Interact CardioVasc Thorac Surg 2019;28:214-21.

Cite this article as: Van Linden A, Hecker F, Courvoisier DS, Arsalan M, Köhne J, Brei C, Holubec T, Walther T. Reduction of drainage-associated complications in cardiac surgery with a digital drainage system: a randomized controlled trial. J Thorac Dis 2019;11(12):5177-5186. doi: 10.21037/jtd.2019.12.20
23. Goh SSC, Chan J, Deng J, et al. Emergency Chest ReExploration in the Intensive Care Unit After Cardiac Surgery: The Geelong Experience. Heart Lung Circ 2013;22:471.

24. Pompili C, Brunelli A, Salati M, et al. Impact of the learning curve in the use of a novel electronic chest drainage system after pulmonary lobectomy: a casematched analysis on the duration of chest tube usage. Inter Cardiovasc Thorac Surg 2011;13:490-3; discussion 493.

25. Linder A, Ertner C, Steger V, et al. Postoperative chest tube management: snapshot of German diversity. Interact Cardiovasc Thorac Surg 2012;15:622-6.

26. Zhou J. Digital chest drainage is better than traditional chest drainage following pulmonary surgery: A metaanalysis. Eur J Cardiothorac Surg 2018;54:635.

27. Batchelor TJP, Rasburn NJ, Abdelnour-Berchtold E, et al. Guidelines for enhanced recovery after lung surgery: recommendations of the Enhanced Recovery After Surgery (ERAS®) Society and the European Society of Thoracic Surgeons (ESTS). Eur J Cardiothorac Surg 2019;55:91-115.

28. Mesa-Guzman M, Periklis P, Niwaz Z, et al. Determining optimal fluid and air leak cut off values for chest drain management in general thoracic surgery. J Thorac Dis 2015;7:2053-7.

29. Ramos Dos Santos PM, Ricci NA, Suster ÉAB, et al. Effects of early mobilisation in patients after cardiac surgery: a systematic review. Physiotherapy 2017;103:1-12.

30. Tschudin-Sutter S, Meinke R, Schuhmacher H, et al. Drainage days-an independent risk factor for serious sternal wound infections after cardiac surgery: a case control study. Am J Infect Control 2013;41:1264-7. 